\title{
SUPRACRICOID PARTIAL LARYNGECTOMY AS SALVAGE SURGERY FOR RADIATION THERAPY FAILURE
}

\author{
Alberto Deganello, MD, ${ }^{1}$ Oreste Gallo, MD, ${ }^{1}$ Jano Maria De Cesare, MD, ${ }^{1}$ Maria Benedetta Ninu, MD, ${ }^{1}$ \\ Gianni Gitti, MD, ${ }^{1}$ Luca de' Campora, MD, ${ }^{2}$ Marco Radici, MD, ${ }^{2}$ Enrico de' Campora, MD ${ }^{1}$ \\ ${ }^{1}$ Department of Otolaryngology-Head and Neck Surgery, University of Florence, Florence, Italy. \\ E-mail: adeganello@hotmail.com \\ ${ }^{2}$ Department of Otolaryngology-Head and Neck Surgery, Fatebenefratelli Hospital, Rome, Italy
}

Accepted 14 January 2008

Published online 4 June 2008 in Wiley InterScience (www.interscience.wiley.com). DOI: 10.1002/hed.20837

\begin{abstract}
Background. The main concern in the treatment of laryngeal carcinomas is tumor control with preservation of laryngeal functions. We believe that salvage supracricoid partial laryngectomy (SPL) should be carefully considered in selected cases of radiotherapy failure, because it can offer the possibility of achieving adequate tumor control with preservation of laryngeal functions.

Methods. A series of 31 patients who underwent an SPL as salvage procedure after radiotherapy failure was reviewed.

Results. Locoregional control rate was $75 \%$, with $60 \%$ 5year overall survival; no patients were lost to follow-up, and a death-from-disease rate of $19.35 \%$ was recorded. Restoration of laryngeal functions was achieved in $89.29 \%$ of the patients. No statistically significant differences were found in locoregional control regarding anterior commissure involvement, elective neck dissection versus wait-and-see policy, pathologic positive neck disease, and restage I-II versus restage III-IV.

Conclusion. The oncologic and functional results indicate the consistency of salvage SPL, proposing this type of operation as a serious alternative to total laryngectomy in carefully selected cases. (C2008 Wiley Periodicals, Inc. Head Neck 30: 1064-1071, 2008
\end{abstract}

Keywords: partial laryngectomy; salvage surgery; larynx carcinoma; radiation failure; neck dissection

Correspondence to: A. Deganello

๑ 2008 Wiley Periodicals, Inc.
The main concern in the treatment of laryngeal carcinomas is tumor control with preservation of whole laryngeal functions.

Early laryngeal cancer can be effectively treated either by larynx preserving surgery or with external beam radiation therapy; results in terms of tumor control and preservation of function for both modalities are satisfactory with reported rates of recurrence or persistence of tumor varying from $10 \%$ to $35 \%{ }^{1-6}$

On the other hand, if a local recurrence occurs after radiation therapy, then surgery is mandatory and remains the only available tool to achieve local control. Total laryngectomy is still considered in most centers the standard treatment for radiotherapy failures. Nevertheless, in selected cases, partial surgery with the attempt to preserve laryngeal function can be considered.

Recent reports in the literature did put in evidence that postradiotherapy supracricoid partial laryngectomy (SPL) is a valid alternative to total laryngectomy in selected cases. ${ }^{7-11}$ SPL can be a safe option from the oncological point of view and, at the same time, is a valuable solution from the functional point of view, offering to patients a chance to control the disease and to retain the 
laryngeal functions even in radiation therapy failures, so that those patients will not experience the full range of functional impairments that follow total laryngectomy.

To understand if salvage SPL represents a valid alternative to total laryngectomy in selected cases, we critically reviewed our direct experience on this subject by means of a retrospective analysis of a 20 -year period.

This paper presents a series of 31 radiation failures treated with SPL in 2 Italian institutions, particularly focusing on the oncological and functional results.

\section{MATERIALS AND METHODS}

From a joined database of the Otolaryngology Department of the University of Florence and the Otolaryngology Department of the Hospital "Fatebenefratelli" in Rome, accounting for 289 patients who underwent a salvage surgical procedure between June 1986 and January 2005 for laryngeal recurrence following elective radiotherapy treatment, the authors identified 31 consecutive patients $(10.73 \%)$ who underwent SPL as a salvage procedure. The remaining 258 patients were treated with different modalities: 226 total laryngectomies $(78.20 \%), 12$ cordectomies in laryngofissure $(4.15 \%), 12$ transoral endoscopic laser excisions $(4.15 \%), 4$ endoscopic nonlaser resections $(1.38 \%)$, and 4 fronto-lateral laringectomies $(1.38 \%)$.

The series included 29 men and 2 women, with age varying between 40 and 72 years (mean, 60.1 years).

Pretreatment TNM staging was corrected using the 20026 th edition ${ }^{12}$ based on the clinical records available.

An overview of the clinical series is shown in Table 1.

Before treatment with radiotherapy, 2 patients were initially seen with Tis glottic lesion, 11 with T1a carcinoma, 12 with T1b, 4 with T2 glottic cancer, and 2 with T2 supraglottic carcinoma. Disease in all patients was clinically classified as N0, so that the series had 2 patients with stage 0 disease, 23 patients with stage I, and 6 patients with stage II. All tumors were biopsy-proven squamous cell carcinomas.

Patients were treated with curative radiation therapy; the total laryngeal dose per patient ranged from 65 to 70 Gy (mean, 66 Gy) fractionated over a period of 6 to 7 weeks. In the 6 patients seen with T2 laryngeal carcinoma, the clinical target volume was extended to include neck lymphatic areas of levels II-IV with a dose of 50 Gy.

Disease-free interval ranged between 2 and 47 months (mean, 13 months); 3 patients were seen with a recurrence within the first 4 months (2 of them showed a recurrence after only 2 months, which probably was actually a persistence of the disease more than a true recurrence), 12 patients experienced a recurrence between 5 and 12 months, 12 patients between 13 and 24 months, 4 patients after 2 years.

All radiotherapy failures were biopsy proven; the extension of the disease was assessed by contrast-enhanced CT scan of the neck and by direct microlaryngoscopy with rigid telescopes at $0^{\circ}, 30^{\circ}$, and $70^{\circ}$ under general anesthesia. Pulmonary metastases were ruled out with thoracic CT scan; pulmonary and cardiac functional tests were satisfactory in all cases.

In 10 patients with recurrent tumors, the restaging after radiotherapy was unchanged, while in the remaining 21 patients an upstaging was recorded (67\%): in 2 patients we had a progression from stage 0 to stage II, in 10 patients from stage I to stage II, in 7 patients from stage I to stage III, and in 2 patients from stage II to stage III.

Institutional Review Board allowance was requested and obtained by the local committee. All 31 patients agreed to the procedure and signed an informed consent form. SPL with cricohyoidpexy (CHP) was performed in 23 cases while the remaining 8 patients underwent SPL with cricohyoidepiglottopexy (CHEP).

Neck dissection was performed in 16 cases, homolateral elective selective neck dissection (SND) of levels II-IV was performed in 11 N0 patients, a therapeutic homolateral functional neck dissection of levels II-V was performed in 5 patients clinically $\mathrm{N} 1$, and in 1 of them, who was seen with a rT3N1 tumor with supraglottic extension, a contralateral SND II-IV was carried out.

Charts were reviewed with particular attention to clinical conditions considered to have an impact in terms of prognosis and restoration of function. Initial clinical tumor stage before radiotherapy, field of irradiation, clinical and pathologic restage of the recurrence, anterior commissure involvement, thyroid cartilage infiltration at pathologic examination, supraglottic extension, elective neck dissection, neck disease, arytenoid involvement, and type of supracricoid surgery (CHP vs CHEP) were tested for impact on loco- 
Table 1. Overview of the clinical series.

\begin{tabular}{|c|c|c|c|c|}
\hline cTNM & rTNM & rpTNM & $\begin{array}{c}\text { Surgery and neck } \\
\text { dissection type (if present) }\end{array}$ & Results \\
\hline T1b No & T2 No & T2 No & CHEP + SND (II-IV) & NED at $41 \mathrm{mo}$ \\
\hline T1a N0 & T2 NO & T2 No & CHEP + SND (II-IV) & NED at $54 \mathrm{mo}$ \\
\hline T2 No & T3 N1 & T3 N1 & $\mathrm{CHP}+\mathrm{FND}(\mathrm{II}-\mathrm{V})$ & NED at $23 \mathrm{mo}$ \\
\hline T1a No & T3 N1 & T3 N1 & $\mathrm{CHP}+\mathrm{FND}(\mathrm{II}-\mathrm{V})$ & NED at $23 \mathrm{mo}$ \\
\hline Tis NO & T2 No & T3 N1 & $\mathrm{CHP}+\mathrm{SND}(\mathrm{II}-\mathrm{IV})$ & $\begin{array}{l}\text { Arytenoid + esophagus recurrence at } 4 \mathrm{mo} \text {; after TL } \\
\text { with esophagectomy and gastric pull-up, patient died } \\
\text { from pulmonary metastases }\end{array}$ \\
\hline T1b No & T2 No & T4 N1 & CHEP + SND (II-IV) & NED at $35 \mathrm{mo}$ \\
\hline T1b NO & T3 N1 & T4 N1 & $\mathrm{CHP}+\mathrm{FND}(\mathrm{II}-\mathrm{V})+\mathrm{SND}(\mathrm{II}-\mathrm{IV})$ & $\mathrm{NED}$ at $37 \mathrm{mo}$ \\
\hline T1b N0 & T3 NO & T3 NO & $\mathrm{CHP}+\mathrm{SND}(\mathrm{II}-\mathrm{IV})$ & NED at $50 \mathrm{mo}$ \\
\hline T1a No & T2 NO & T2 No & CHEP + SND (II-IV) & NED at $60 \mathrm{mo}$ \\
\hline Tis NO & T2 NO & T2 No & CHEP + SND (II-IV) & NED at $60 \mathrm{mo}$ \\
\hline T2 No & T3 N1 & T4 N1 & $\mathrm{CHP}+\mathrm{FND}(\mathrm{II}-\mathrm{V})$ & Arytenoid recurrence at $9 \mathrm{mo}$; after TL, NED at $48 \mathrm{mo}$ \\
\hline T1a N0 & T3 N1 & T3 N1 & $\mathrm{CHP}+\mathrm{FND}(\mathrm{II}-\mathrm{V})$ & NED at $60 \mathrm{mo}$ \\
\hline T1b No & T2 NO & T2 No & $\mathrm{CHP}+\mathrm{SND}(\mathrm{II}-\mathrm{IV})$ & NED at $45 \mathrm{mo}$ \\
\hline T1b NO & T2 NO & T2 No & $\mathrm{CHP}+\mathrm{SND}(\mathrm{II}-\mathrm{IV})$ & NED at $41 \mathrm{mo}$ \\
\hline T1a No & T2 NO & $\mathrm{T} 2 \mathrm{NO}$ & $\mathrm{CHP}+\mathrm{SND}(\mathrm{II}-\mathrm{IV})$ & NED at $30 \mathrm{mo}$ \\
\hline T1b No & T3 NO & T3 N1 & $\mathrm{CHP}+\mathrm{SND}(\mathrm{II}-\mathrm{IV})$ & NED at $24 \mathrm{mo}$ \\
\hline T1a N0 & T3 NO & T3 NO & $\mathrm{CHP}$ & NED at $37 \mathrm{mo}$ \\
\hline T2 No & T2 NO & T2 No & $\mathrm{CHP}$ & NED at $80 \mathrm{mo}$ \\
\hline T1b NO & T2 NO & T2 No & $\mathrm{CHP}$ & $\begin{array}{l}\text { Cricoid recurrence after } 4 \text { mo; TL performed but patient } \\
\text { dies after another } 2 \text { mo }\end{array}$ \\
\hline T1a No & T1b N0 & T1b No & $\mathrm{CHP}$ & $\begin{array}{l}\text { Second T3 cancer of the piriform sinus after } 43 \mathrm{mo} \text {; total } \\
\text { pharyngolaryngectomy performed with RT. Patient } \\
\text { dies from unresectable neck metastases after } 62 \text { mo }\end{array}$ \\
\hline T1a No & T2 No & T2 No & $\mathrm{CHP}$ & $\begin{array}{l}\text { Patient develops neck metastases after } 6 \text { mo and } \\
\text { receives neck dissection with postoperative RT. After } \\
12 \text { mo, patient dies from a new neck recurrence }\end{array}$ \\
\hline T1b N0 & T2 NO & T1b N0 & $\mathrm{CHP}$ & $\begin{array}{l}\text { Patient develops neck metastases after } 6 \mathrm{mo} \text { and } \\
\text { treated with chemo. Patient dies after } 20 \mathrm{mo}\end{array}$ \\
\hline T1b No & T1b N0 & T1b N0 & $\mathrm{CHP}$ & NED at $65 \mathrm{mo}$ \\
\hline T1a No & T1b N0 & T1b No & CHEP & NED at $30 \mathrm{mo}$ \\
\hline T1a N0 & T3 NO & T3 NO & $\mathrm{CHP}$ & $\begin{array}{l}\text { Patient receives total laryngectomy for an ab ingestis } \\
\text { pneumonia. NED at } 60 \text { mo }\end{array}$ \\
\hline T2 No & T2 No & T2 No & $\mathrm{CHP}$ & NED at $85 \mathrm{mo}$ \\
\hline T1b N0 & T1b N0 & T1b N0 & CHEP & NED at $60 \mathrm{mo}$ \\
\hline T2 No & T2 NO & T2 No & $\mathrm{CHP}$ & NED at $41 \mathrm{mo}$ \\
\hline T1a No & T1a No & T2 No & $\mathrm{CHP}$ & Patient dies after 50 mo from vesical cancer \\
\hline T1b No & T1b NO & T1b No & $\mathrm{CHP}$ & NED at $44 \mathrm{mo}$ \\
\hline T2 No & T2 No & T2 No & CHEP & $\begin{array}{l}\text { Tracheal recurrence at } 14 \mathrm{mo} \text {; treated with chemo. } \\
\text { Patient dies after } 19 \mathrm{mo}\end{array}$ \\
\hline
\end{tabular}

Abbreviations: CTNM, clinical TNM staging of the original neoplasia, prior to radiotherapy: rTNM. clinical TNM staging of the postradiotherapy relapse, prior to salvage surgery; rDTNM, histopathologic TNM staging of the postradiotherapy relapse; CHEP, crico hyoid epiglotto pexy; SND, selective neck dissection (followed by the resected levels); NED, no evidence of disease; CHP, crico hyoid pexy; FND, functional neck dissection (followed by the resected levels); $T L$, total laryngectomy; $R T$, radiotherapy; chemo, chemotherapy.

regional control. Arytenoid resection, a type of supracricoid surgery (CHP vs CHEP), and the adjunction of elective neck dissection were tested in terms of functional results based upon time of decannulation and time of oral intake restoration.

The statistical analysis was performed with an IBM computer using STATA (Stata Corporation, College Station, TX).

Considering the day of the salvage surgery as the starting day of the observation, follow-up was for a minimum of 2 years or until death (mean, 45 months; minimum 6, maximum 180). Follow-up end points included evidence of local or regional recurrence, death from disease, and death from other causes. No patients were lost to follow-up.

Overall survival was calculated using the Kaplan-Meier method, considering the day of salvage partial laryngectomy as the starting day of the observation and death from any cause as the endpoint. Locoregional control was calculated using the Kaplan-Meier method, considering the day of salvage partial laryngectomy as the start- 


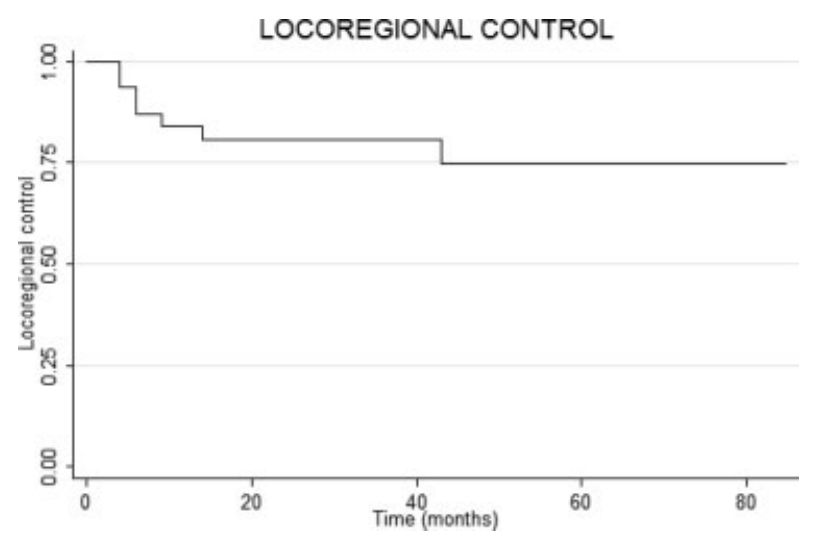

FIGURE 1. Kaplan-Meier 5-year locoregional control for salvage supracricoid partial laryngectomy; falls in the curve represent appearance of relapse. Seventy-five percent of the patients were disease-free at the end of the observation.

ing day of the observation and the reoccurrence of local or neck disease as endpoint. The impact of the reviewed clinical features in terms of locoregional control was analyzed using the KaplanMeier curves; the difference between groups was compared using the log-rank test. Probability values less than .05 were considered statistically significant.

\section{RESULTS}

The pathologic examination showed a further upstaging in 5 cases, a downstaging in 1 case, and a pathologic confirmation of the clinical restaging for the remaining 25 patients.

An overall complication rate of $16.13 \%$ was recorded. No perioperative or postoperative mortality occurred, but major complications occurred in 3 cases $(9.67 \%)$ : 1 patient, who had been treated with a CHP, had to undergo a total laryngectomy because of severe postoperative aspiration, and no tumor was found in the specimen; in 2 other patients, the development of a salivary fistula required a surgical revision, with direct closure of the pharyngeal opening in 1 case and with closure with a pectoralis major flap in the other case; in both patients the larynx was preserved. Minor complications occurred in 2 patients $(6.45 \%)$ that suffered of a cervical wound infection that healed with medical care.

In the series, 3 patients underwent a totalization of the laryngectomy because of oncologic reasons (see Table 1), so that functional results were calculated among the remaining 28 patients: in 25 , a complete restoration of laryngeal function was achieved (89.29\%), 1 patient received a total laryngectomy because of aspiration pneumonia, in 1 case decannulation never succeeded, and 1 patient had to retain a permanent gastrostomy to compensate unsatisfactory oral intake.

The mean time for decannulation was 27 days (range, 14-59 days), and the mean time for oral intake restoration was 30 days (range, 12-72 days). The frequency of arytenoid resection is often felt to be an important factor in the restoration of function; in our series, we resected 1 arytenoid in 11 cases, while both arytenoids were preserved in the remaining 20 patients. No differences in terms of decannulation or restoration of oral intake were noticed between the 2 groups; however, we did identify a subgroup of patients (6 cases) in which we recorded a prolonged decannulation time (mean value, 40 days) with normal oral food intake restoration time. These 6 patients received a CHEP with preservation of both arytenoids. Other factors were evaluated in terms of restoration of laryngeal function, and no differences were noticed related to type of laryngectomy (CHP vs CHEP) and no neck dissection versus monolateral or bilateral neck dissection.

According to the Kaplan-Meier method, locoregional control rate was $75 \%$, with $60 \% 5$ year overall survival percentage (Figures 1 and 2).

No statistically significant differences $(p>.05)$ were found in locoregional control with regard to anterior commissure involvement, elective neck dissection versus wait-and-see policy, pathologic positive neck disease, restage I-II versus restage III-IV, thyroid cartilage infiltration at pathologic examination, supraglottic tumor extension, arytenoid involvement, and type of supracricoid sur-

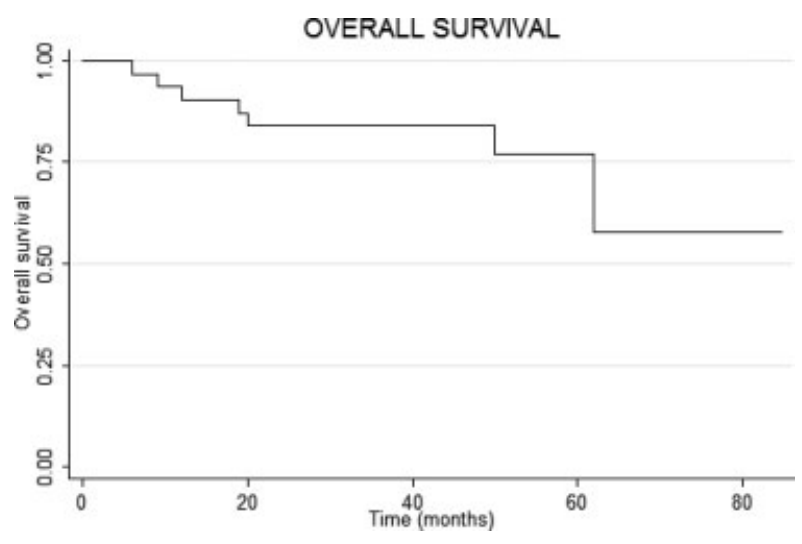

FIGURE 2. Kaplan-Meier 5-year overall survival for salvage supracricoid partial laryngectomy. Falls in the curve reflect deaths by any cause during the observation period. Sixty percent of patients lived past the end of the follow-up time. 

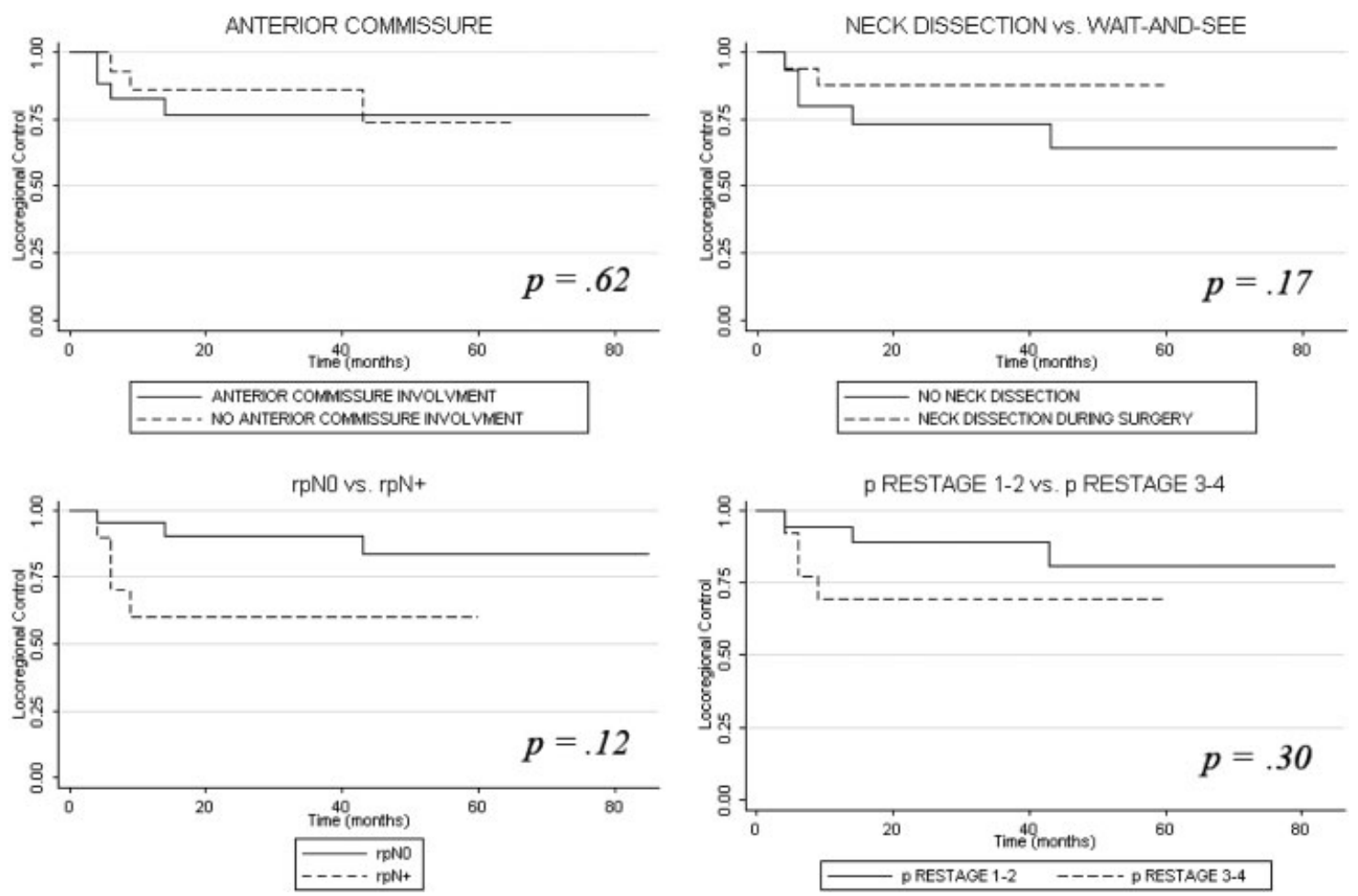

FIGURE 3. No statistical significance was found in locoregional control regarding anterior commissure involvement, neck dissection, pathological nodal status, or advanced restage. Endpoint of observation was Jan 2005.

gery (CHP vs CHEP). In Figure 3, we present the most significant ones.

Concerning neck results in the 31 patients with recurrences that had been originally staged N0 before radiotherapy, 5 patients were seen with a N1 neck disease, whereas the remaining 26 were restaged N0. Neck dissection was performed in the $5 \mathrm{~N}+$ patients and in $11 \mathrm{~N} 0$ patients, whereas 15 patients were admitted to a wait-andsee policy. Occult neck metastasis at pathologic examination of the specimen was found in 3 cases; furthermore, in the wait-and-see group, 2 patients developed a recurrence in the neck without any evidence of local relapse or second primaries, so we can suppose that they were seen with an occult neck disease at the time of surgery, which subsequently turned into a clinical neck recurrence. Thus, in our series, the rate of occult neck metastasis in patients with N0 recurrent tumors after radiotherapy is 5 of $26(19.23 \%)$ (Figure 4$)$.

A locoregional failure occurred in 7 patients (Table 1).

Four patients were seen with a local relapse, 1 patient developed a second primary after 4 years, and the remaining 2 patients had a relapse in the neck.
A second salvage surgery was attempted in 5 of 7 failures: 2 patients underwent a total laryngectomy, 1 patient had total laryngectomy with total pharyngectomy, 1 patient a total laryngectomy with total pharyngectomy and total esophagectomy, and 1 patient had a neck dissection with postoperative radiation on the neck.

The second salvage surgery was effective only in 1 case in which a local recurrence at the arytenoid level was successfully treated with total

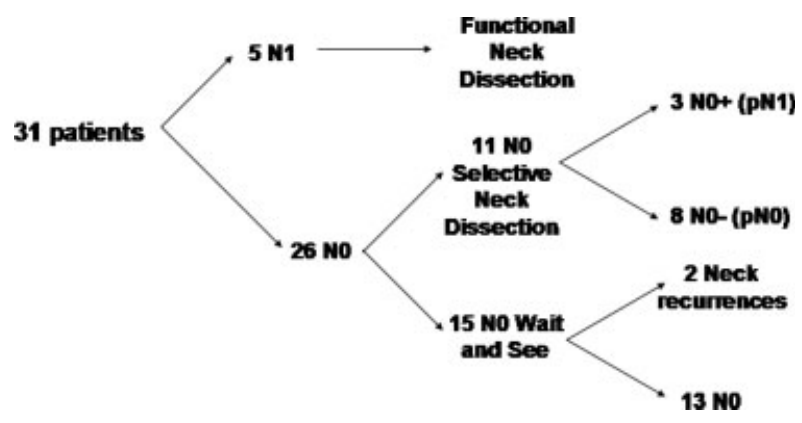

FIGURE 4. Overview of the clinical series for what concerns neck findings. Among 26 NO patients, we found occult neck disease in 3 patients of the electively dissected group and 2 neck recurrences developed in the wait-and-see group: $19 \%$ occult neck disease rate. 
laryngectomy, and for the remaining 4 cases the mean survival time after the second salvage procedure was 10 months.

\section{DISCUSSION}

The optimal management of a laryngeal recurrence after radiotherapy should guarantee a safe tumor clearance with organ preservation that enables the patients to preserve speech, to allow a normal oral alimentation, and to avoid a permanent tracheostomy.

As a general rule, it has been for a long time postulated that in a previously irradiated larynx, if a partial laryngectomy is attempted, the incidence of surgical complications is extremely high, so that any form of laryngeal preserving operation should be discouraged. This rule arises from the evidence that the irradiated laryngeal tissues, and almost the entire laryngeal cartilaginous framework, eventually suffer from a consistent fibrosis and hypovascularization that would prevent the possibility of performing any sort of partial laryngectomy without risking a fearsome condritis, the occurrence of salivary fistulas, pexy disruption, or other local infection. All these complications will in most cases result in an important detriment of the laryngeal functions so that in most cases a total laryngectomy has to be performed anyhow. ${ }^{13}$

Our series of 31 patients accounts for the $10.73 \%$ of the 289 surgical salvage procedures for radiotherapy laryngeal failures performed in the institutions conducting the study. However, if we look at the distribution in time of salvage SPL, it is interesting to notice that during the first 10 years of observation (period: 1986-1995) over a total of 160 radiotherapy failures, $134(83.75 \%)$ received a salvage total laryngectomy, $10(6.25 \%)$ a salvage SPL, and $16(10 \%)$ patients were treated with other surgical procedures; in the following 10 years of observation (period: 1996-2005) over a total of 129 radiotherapy failures, $92(71.31 \%)$ received salvage total laryngectomy, 21 (16.27\%) a salvage SPL, and $16(12.40 \%)$ were treated with other surgical procedures. These data reflect that, even if salvage SPL still remains indicated only for a minority of cases, its frequency has more than doubled in the last 10 years.

Total laryngectomy for salvage in cases of early laryngeal cancers after radiotherapy achieves local control in $78 \%$ to $85 \%$ of cT1 (preradiotherapy staging) and $65 \%$ of cT2 (preradiotherapy staging) glottic failures, and $40 \%$ to $55 \%$ of cT $1-2$ (preradiotherapy staging) supraglottic irradiation failures, ${ }^{14-18}$ but total laryngectomy is not free from complications. Pharyngocutaneous fistulas, wound infections, chyle leak, swallowing, and air problems have been reported as complications of total laryngectomy, with overall complications rates of $20 \%$ to $40 \% .{ }^{19,20}$ The incidence of pharyngocutaneous fistula is reported to be as high as $15 \%$ to $20 \%$, while that of severe complications (carotid rupture, major wound breakdown, severe pharyngeal-esophageal stricture) is $10 \% .^{20,21}$

We must consider, however, that in the group of salvage total laryngectomies, surgeons deal with more locoregionally extended recurrences that require extensive demolitions, and often the recurrent disease presents after chemoradiation, which significantly increases the rate of surgical complications. ${ }^{19}$

If the recurrent tumor is locally limited, a transoral $\mathrm{CO}_{2}$ laser excision could be a valid option in experienced hands ${ }^{22}$; however, local control rate after 1 surgical session is reported by Steiner et al to be only of $38 \%$ and local control of $71 \%$ is reached after repeated laser surgery procedures.

In two-thirds of the cases of our series, a recurrence was seen with a more advanced stage than the original tumor; however, all of them were suitable for a salvage SPL. Clinical restaging of recurrent tumors is particularly difficult, and a precise and careful assessment by means of endoscopy and imaging is required.

General and specific indications and contraindications for SPL as salvage surgery do not differ from those in nonirradiated tumors and are already mentioned in other papers. ${ }^{13,23}$ Selection is the key to success, and the patient should be informed about the possibility of an intraoperative conversion of the partial laryngectomy into a total laryngectomy for oncological reasons. Submucosal subtle tumor spread is a fearsome clinical feature of recurrent squamous cell carcinomas after radiotherapy so that a meticulous assessment of the key points (crico-arytenoid joint, interarytenoid tissue, subglottic margin) with frozen sections during surgery is mandatory. Furthermore, we believe that if the definitive pathologic examination of the specimen does not comport with adequate tumor radicality, a completion of the laryngectomy is advisable.

The overall complication rate in this series $(16.13 \%)$ is acceptable, demonstrating that SPL as salvage surgery is feasible. 
We believe that some technical accuracy during surgery is helpful to decrease postoperative complications. After elevation of the subplatysma apron flap, we routinely suture a compress soaked with cool Ringer solution to cover the entire inner surface of the skin flap to prevent vascular problems caused by excessive drying of irradiated tissues. Furthermore, it is important to detach the infrahyoid muscles from the hyoid bone in an "en bloc" manner so that the entire bloc of the strap muscles together with the thyroid isthmus is separated from the larynx and dissected downward, respecting as much as possible the vascular supply coming from the superior and inferior thyroid arteries and veins. This surgical step does not interfere with the radicality of the neck dissection. These infrahyoid musculo-glandular structures will provide an enforcement cover to the pexy with the intent to prevent disruptions.

The fact that the entire thyroid cartilage (with the only exception of the inferior cornua to protect the recurrent laryngeal nerves) is removed decreases the incidence of condritis when compared with salvage vertical partial laryngectomies or salvage supraglottic laryngectomies. In SPL, the remaining cartilages are the cricoid and 1 or both arytenoids that should not be subpericondrially exposed.

Pellini et al did analyze postoperative complications and functional results, comparing 65 patients treated with SPL with 17 patients who received salvage SPL for radiotherapy failure. They found no statistically significant differences between the 2 groups in terms of postoperative complications, decannulation time, and restoration of oral intake. ${ }^{24}$

In our series, functional results were subjectively evaluated: in $89.29 \%$ of the cases, decannulation, restoration of oral intake, and satisfactory voice production that allowed normal social interactions were achieved.

In 2002, Rifai et al ${ }^{25}$ presented a series of 37 radiotherapy failures that were treated with a supracricoid laryngectomy (either CHP or CHEP) and stated that preservation of both arytenoids made recovery of laryngeal function much easier.

In our series, we found no differences in terms of time of decannulation and oral intake restoration between patients with both arytenoids preserved versus those with 1 residual arytenoid. These functional results highlight the possibility of resecting 1 arytenoid for oncologic reasons without jeopardizing oral intake restoration. It is interesting to notice that the preservation of both arytenoids while performing a CHEP has been found to be related to prolonged decannulation time, which might be connected with edema of both arytenoids and of the residual epiglottis resulting in transient laryngeal inlet stenosis.

Our functional results match data reported in literature, in which functional outcome of SPL seems not to differ in irradiated patients compared with previously untreated cases.

Furthermore, we did not record a higher incidence of complications and functional problems in the group that underwent a neck dissection when compared with the wait-and-see group.

The oncologic results, with $75 \%$ of locoregional control rate and an organ preservation rate of $90 \%$, indicate the consistency of salvage SPL, proposing this type of operation as a serious alternative to total laryngectomy in carefully selected cases. A critical analysis of our series reveals that 6 patients (19.35\%) did die of cancer relapse (see Table 1): among them 2 patients died from neck disease, 1 patient developed a second primary hypopharyngeal carcinoma at 43 months from salvage SPL, and the remaining 3 patients $(9.68 \%)$ died of a local relapse and might have benefited from a salvage total laryngectomy instead of the SPL they received.

The role of elective neck treatment in conservative laryngeal salvage surgery after radiotherapy for N0 recurrent tumors is still controversial.

In our series, the criteria to perform an elective neck treatment were mostly subjective so that we tried to gain from our data some helpful information for future treatment strategies; we had a $67 \%$ rate of upstaged recurrences, occult neck disease rate was found to be $19.23 \%$ and always in upstaged recurrences.

To understand the impact of elective neck radiation therapy on neck relapses, we looked at the radiation therapy field. The initial radiotherapy field in the $5 \mathrm{~N} 0+$ recurrences included the larynx only ( 1 patient had Tis, 1 had T1a, and 3 had T1b), for what concerns the restaging all 5 of them showed an upstaged laryngeal cancer (4 patients turned into a rT2 and 1 turned into a rT3).

We also looked at the $6 \mathrm{~T} 2$ cancers in which the radiation field included levels II-IV: all of them were again restaged as T2, and 2 of them were seen with N1 neck disease despite elective neck irradiation. This suggests that, despite an adequate elective radiation in the neck, the radiotherapy does not prevent neck metastasis in recurrent $\mathrm{T} 2$ tumors, even if in our series these metastases were all clinically evident and no 
occult neck disease developed in the remaining 4 N0 patients that were admitted to a wait-and-see protocol.

Locoregional control did not differ statistically between the 2 groups (wait-and-see and electively dissected group); however, we did not experience a higher complication rate in the dissected group, and, furthermore, in the wait-and-see group, we were unable to control 2 relapses in the neck. These findings suggest that in T2-3 upstaged recurrences, even if the neck was electively irradiated, an SND of level II-IV is advisable.

\section{CONCLUSION}

SPL is a feasible, oncologically safe, functionally valid alternative to total laryngectomy in selected laryngeal recurrences after radiotherapy.

The overall complication rate in this series is comparable to that with other salvage surgical procedures. Selection is the key to success, and the conversion to a total laryngectomy has to be done whenever any doubt exists about the adequacy of resection margins or if required by serious functional problems. Ipsilateral elective neck dissection of levels II-IV is advisable in T2-3 upstaged recurrences. Prognosis of second recurrences after salvage surgery remains poor even after adequate surgical resection.

\section{REFERENCES}

1. de' Campora E, Radici M, de' Campora L. External versus endoscopic approach in the surgical treatment of glottic cancer. Eur Arch Otorhinolaryngol 2001;258:533536.

2. de' Campora E, Radici M, de' Campora L. Glottic-hypoglottic laryngectomy in the treatment of laryngeal tumors. Acta Otorhinolaryngol Ital 2001;21:151-155.

3. de' Campora E. Partial vertical laryngectomy (anterior frontal laryngectomy, fronto-lateral laryngectomy). Indications and long-term results. Acta Otorhinolaryngol Ital 1991;11(Suppl 33):39-44.

4. Small W Jr, Mittal BB, Brand WN, et al. Results of radiation therapy in early glottic carcinoma: multivariate analysis of prognostic and radiation therapy variables. Radiology 1992;183:789-794.

5. Goor KM, Peeters AJ, Mahieu HF, et al. Cordectomy by $\mathrm{CO}(2)$ laser or radiotherapy for small T1a glottic carcinomas: costs, local control, survival, quality of life, and voice quality. Head Neck 2007;29:128-136.

6. Jose B, Calhoun DL, Mohammed A. Recurrences after irradiation in early vocal cord cancer with literature review. J Surg Oncol 1984;27:224-227.
7. Spriano G, Pellini R, Romano G, Muscatello L, Roselli R. Supracricoid partial laryngectomy as salvage surgery after radiation failure. Head Neck 2002;24:759-765.

8. Yiotakis J, Stavroulaki P, Nikolopoulos T, et al. Partial laryngectomy after irradiation failure. Otolaryngol Head Neck Surg 2003;128:200-209.

9. Clark J, Morgan G, Veness M, et al. Salvage with supracricoid partial laryngectomy after radiation failure. ANZ J Surg 2005;75:958-962.

10. Laccourreye O, Weinstein G, Naudo P, et al. Supracricoid partial laryngectomy after failed laryngeal radiation therapy. Laryngoscope 1996;106:495-498.

11. Makeieff M, Venegoni D, Mercante G, et al. Supracricoid partial laryngectomies after failure of radiation therapy. Laryngoscope 2005;115:353-357.

12. Sobin LH, Wittekind Ch, editors. TNM classification of malignant tumors, 6th ed. International Union Against Cancer. New York: Wiley-Liss; 2002.

13. Bradley PJ, Ferlito A, Suarez C, et al. Options for salvage after failed initial treatment of anterior vocal commissure squamous carcinoma. Eur Arch Otorhinolaryngol 2006;263:889-894.

14. Spriano G, Antognoni P, Piantanida R, et al. Conservative management of T1-T2N0 supraglottic cancer: a retrospective study. Am J Otolaryngol 1997;18:299-305.

15. DeSanto LW, Lillie JC, Devine KD. Surgical salvage after radiation for laryngeal cancer. Laryngoscope 1976; 86:649-657.

16. Ballantyne AJ, Fletcher GH. Surgical management of irradiation failures of nonfixed cancers of the glottic region. Am J Roentgenol Radium Ther Nucl Med 1974; 120:164-168.

17. Hawkins NV. The treatment of glottic carcinoma: an analysis of 800 cases. Laryngoscope 1975;85:1485-1493.

18. Schwaab G, Mamelle G, Lartigau E, et al. Surgical salvage treatment of $\mathrm{T} 1 / \mathrm{T} 2$ glottic carcinoma after failure of radiotherapy. Am J Surg 1994;168:474-475.

19. Ganly I, Patel SG, Matsuo J, et al. Results of surgical salvage after failure of definitive radiation therapy for early-stage squamous cell carcinoma of the glottic larynx. Arch Otolaryngol Head Neck Surg 2006;132:59_ 66.

20. Ganly I, Patel S, Matsuo J, et al. Postoperative complications of salvage total laryngectomy. Cancer 2005;103: 2073-2081.

21. Parsons JT, Mendenhall WM, Stringer SP, et al. Salvage surgery following radiation failure in squamous cell carcinoma of the supraglottic larynx. Int $\mathrm{J}$ Radiat Oncol Biol Phys 1995;32:605-609.

22. Steiner W, Vogt P, Ambrosch P, Kron M. Transoral carbon dioxide laser microsurgery for recurrent glottic carcinoma after radiotherapy. Head Neck 2004;26:477484.

23. Laccourreye H, Laccourreye O, Weinstein G, et al. Supracricoid laryngectomy with cricohyoidopexy: a partial laryngeal procedure for selected supraglottic and transglottic carcinomas. Laryngoscope 1990;100:735741.

24. Pellini R, Manciocco V, Spriano G. Functional outcome of supracricoid partial laryngectomy with cricohyoidopexy: radiation failure vs previously untreated cases. Arch Otolaryngol Head Neck Surg 2006;132:1221-1225.

25. Rifai M, Heiba MH, Salah H. Anterior commissure carcinoma. II. The role of salvage supracricoid laryngectomy. Am J Otolaryngol 2002;23:1-3. 